\title{
Características Sociodemográficas y Clínicas de los Pacientes Tratados por Primera Vez por Cáncer Escamocelular Oral. Medellín, Colombia
}

\author{
Sociodemographic and Clinic Characteristics of the Patients Threatened \\ at First Time for Squamous Cell Oral Cancer. Medellín, Colombia
}

\author{
Adriana Posada-López'; Marta Aída Palacio-Correa² \& Andrés A. Agudelo-Suárez
}

POSADA-LÓPEZ, A.; PALACIO-CORREA, M. A. \& AGUDELO-SUÁREZ, A. A. Características Sociodemográficas y clínicas de los pacientes tratados por primera vez por cáncer escamocelular oral. Medellín, Colombia. Int. J. Odontostomat., 12(3):237-245, 2018.

RESUMEN: Este estudio pretendió describir las características sociodemográficas y clínicas de los pacientes tratados por primera vez por cáncer escamocelular oral (CEB) en Medellín (Colombia). Para ello se realizó un estudio descriptivo anidado en una cohorte dinámica retrospectiva de casos de CEB, procedente de 9 centros oncológicos especializados de la ciudad entre 2000 y 2011, según la información de las historias clínicas y clasificadas según la CIE-10. Se recolectaron variables sociodemográficas y clínicas como: tratamiento recibido, localización del tumor, estadio del tumor, atención recibida (profesional), reporte de consumo de alcohol o cigarrillo, complicaciones asociadas al tratamiento de CEB. Se describieron las variables del estudio y pruebas Chi cuadrado para observar diferencias entre algunas características clínicas y el sexo. Se encontraron 778 casos de CEB en el periodo estudiado, con una edad promedio de diagnóstico de $63,5( \pm 13,6)$ años. Más de la mitad tenían estratos socioeconómicos y niveles educativos bajos. Un $56 \%$ de las mujeres y un $63 \%$ de los hombres fueron diagnosticados en un estadio IV del tumor. Los tratamientos más realizados fueron de tipo combinado o cirugía. Un $35 \%$ presentaron problemas cardiovasculares, aunque no se reportaron diferencias estadísticamente significativas entre la presencia de determinadas comorbilidades y el sexo. Se reportó el consumo de alcohol en un $19 \%$ y de cigarrillo en un $51 \%$ con mayor consumo en hombres en ambos casos y diferencias estadísticamente significativas $(p<0,0001)$ con respecto a las mujeres. En cuanto a complicaciones asociadas al tratamiento para CEB, se reportaron con mayor frecuencia disfagia ( $47 \%$ ) y mucositis $(24 \%)$. Más de la mitad de los tumores de presentaron en la lengua. Se requieren estrategias que permitan sistemas de vigilancia epidemiológica para el CEB, así como mejorar los programas de prevención y tratamiento precoz para esta enfermedad en consonancia con las políticas y los planes nacionales y globales.

PALABRAS CLAVE: carcinoma de células escamosas, neoplasias de la boca, perfil epidemiológico, vigilancia epidemiológica.

\section{INTRODUCCIÓN}

El cáncer oral se considera una enfermedad de gran impacto en las poblaciones por su magnitud, por que afecta la calidad de vida de las personas que lo padecen y por ser causa de muerte al igual que otros tipos de cánceres (Ferlay et al., 2015). Según datos de la Organización Mundial de la Salud se reportan 529 mil nuevos casos de cáncer de la cavidad oral y faringe cada año, y ocurren más de 300 mil muertes (Ferlay et al.). La tasa de incidencia estandarizada por edad del cáncer oral es de 2,7 por cada 100 mil habitantes y se encuentran diferencias por sexo, edad y región. De igual manera, existen determinantes contextuales de esta enfermedad como por ejemplo el Índice de Desarrollo Humano (IDH). La proporción de incidencia de cáncer de la cavidad oral es más alta en mujeres que proceden de países que reportan un bajo IDH (Shield et al., 2017).

\footnotetext{
${ }^{1}$ Facultad de Odontología. Universidad de Antioquia. Medellín, Colombia.

${ }^{2}$ Facultad de Trabajo Social. Universidad Pontificia Bolivariana. Medellín, Colombia.

Financiación: Grupo de Epidemiología. Facultad Nacional de Salud Pública. Universidad de Antioquia. Medellín, Colombia.
} 
En Colombia, las estadísticas para el cáncer de labios, cavidad oral y faringe no son recientes. Según datos del Instituto Nacional de Cancerología (2017), cuyas estimaciones disponibles son del periodo 2002-2006, se reportaron 768 casos anuales con una tasa de incidencia cruda (TIC) de 3,7 por cada 100 mil habitantes y una tasa de incidencia ajustada por edad (TAE) de 4,5 por cada 100 mil habitantes para el caso de los hombres (para las mujeres se reportaron 798 casos anuales con una TIC de 3,7 y una TAE de 4,2) (Instituto Nacional de Cancerología).

Si bien el cáncer oral puede considerarse un problema de salud pública y de importancia en la agenda científica y política en el país, las investigaciones que caracterizan el problema en Colombia son escasas. Una revisión de la literatura disponible en bases de datos en ciencias de la salud y literatura gris muestra en términos generales, investigaciones de carácter local y muy específicas en términos epidemiológicos y clínicos (Álvarez Gómez et al., 2008; Álvarez Martínez et al., 2010; Álvarez Martínez et al., 2011; Martínez Martínez et al., 2016). Se encontró un estudio a nivel poblacional que analizó las tendencias relacionadas con la incidencia y mortalidad por Cáncer Oral en la ciudad de Santiago de Cali, utilizando los registros poblacionales en el periodo comprendido entre 1962- 2007 (Ordóñez et al., 2014). Por último se reportó un estudio epidemiológico donde se estudia la incidencia de cáncer oral por demanda de atención, entre los años 1989 y 2008, utilizando los reportes del Instituto Nacional de Cancerología (Bernal-Baláez, 2016).

Por tanto, se requieren estrategias que permitan fortalecer los sistemas de vigilancia epidemiológica para disponer de datos epidemiológicos confiables en cáncer oral y establecer herramientas para el diagnóstico oportuno y ofrecer acceso efectivo a los servicios de salud. De ahí se hace importante conocer las características de los pacientes que son atendidos en las instituciones prestadoras de servicios de salud en el país.

De acuerdo a lo anterior se planteó este estudio con el fin de describir las características sociodemográficas y clínicas de los pacientes tratados por primera vez por cáncer escamocelular oral (CEB) en Medellín, Colombia.

\section{MATERIAL Y MÉTODO}

Se trata de un estudio descriptivo anidado en una cohorte dinámica retrospectiva de casos de CEB, tratados por primera vez en 9 centros oncológicos especializados de Medellín entre 2000 y 2011. Para la selección de los casos, se procedió a analizar las historias clínicas de los pacientes diagnosticados con CEB como tumor primario (códigos C00 hasta C06, C10 y C14, según la Clasificación Internacional de Enfermedades versión 10, CIE 10), lo que permitió, con base en la confirmación histopatológica, la elección de los pacientes con CEB y que habían sido tratados por primera vez en los centros oncológicos participantes en el estudio. En total, de las 1700 historias que se revisaron manualmente, 778 pacientes cumplieron los criterios de inclusión y se les realizó el análisis respectivo. Para mayor detalle de otros aspectos relacionados con la metodología, se puede consultar una publicación previa del equipo investigador (Posada-López et al., 2016).

A partir de la información consignada en la historia clínica se diseñó un instrumento de recolección de datos, el cual se mejoró de acuerdo con las sugerencias derivadas de la prueba piloto. Los datos fueron consignados de forma retrospectiva, tomando en cuenta variables epidemiológicas de persona, tiempo, lugar y clínicas. Los sesgos del observador se controlaron con la asesoría de un cirujano oncólogo de cabeza y cuello y un cirujano maxilofacial con experiencia en cáncer oral; los cuales tuvieron un papel importante en el diseño del instrumento, especialmente en lo que se relaciona con las variables clínicas.

Concretamente para este estudio descriptivo se utilizaron las siguientes variables: sexo, edad al momento de diagnóstico de CEB, lugar de residencia, estado civil, nivel socioeconómico, nivel educativo y afiliación al sistema de salud. En cuanto al estrato socioeconómico, en Colombia las viviendas se clasifican en 6 estratos socioeconómicos denominados 1 : bajo-bajo, 2: bajo, 3: medio-bajo, 4: medio, 5: medioalto y 6: alto; de los cuales 1,2 y 3 corresponden a estratos que albergan a los usuarios con menos recursos económicos y son beneficiarios de subsidios, y los estratos 5 y 6 para usuarios con mayores recursos (Departamento Administrativo de Planeación, 2011). El Sistema General de Seguridad Social en Salud funciona en dos regímenes: el contributivo y el subsidiado; las personas con capacidad de pago como los trabajadores, los pensionados y sus familias se 
deben vincular al régimen contributivo y en el subsidiado están aquellos ciudadanos sin capacidad de pago que reciben el servicio de salud mediante un subsidio que ofrece el Estado (Guerrero et al., 2011). Como variables clínicas del estudio se consideraron las siguientes: tratamiento recibido, localización del tumor, estadio del tumor, atención recibida (profesional), reporte de consumo de alcohol o cigarrillo, complicaciones asociadas al tratamiento de CEB.

Se realizó un análisis descriptivo de los datos calculando frecuencias absolutas y relativas según las variables seleccionadas en el estudio en forma general y estratificando por sexo, realizando pruebas de significación estadística tipo Chi cuadrado para observar relaciones entre las características clínicas y el sexo, localización del tumor según sexo y estadio del tumor.

Este estudio cumplió con los requisitos éticos para investigación según la normativa nacional e internacional (Ministerio de Salud y Protección Social, 1993; World Medical Association General Assembly, 2004). Según la Resolución 8430 del Ministerio de Salud este estudio se considera sin riesgo, puesto que se basa en datos secundarios que proceden de historias clínicas. Todos los datos se trataron de forma anónima y confidencial. El proyecto de investigación fue aprobado por el Comité de Bioética de la Facultad Nacional de Salud Pública de la Universidad de Antioquia (Acta 051 de 2011). Adicionalmente los comités de investigación y/o de bioética de las instituciones participantes dieron su aval. El reporte de esta investigación de redactó de acuerdo a la guía STROBE para estudios observacionales (von Elm et al., 2008).

\section{RESULTADOS}

Durante el periodo de estudio, se encontraron 778 casos de cáncer escamocelular oral $(45,5 \%$ mujeres) en las instituciones participantes. De acuerdo a la información de la historia clínica se pudo determinar la edad en el momento del diagnóstico en 556 pacientes, con una edad mínima de 7 años y una edad máxima de 98 . Se observó que la edad promedio al momento del diagnóstico fue $63,5( \pm 13,6)$ años (Hombres: $62,6 \pm 12,5$, Mujeres: $64,5 \pm 14,8 ; p=0,076)$. Sólo el $21,3 \%$ de los pacientes tenían en su historia clínica el tiempo transcurrido entre la aparición o percepción de la lesión y el momento en el cual fue histopatológicamente confirmado el CEB, tiempo que oscila entre 15 días y 96 meses. Adicionalmente, se encontró que para el $50 \%$ de los pacientes transcurrieron 58 días o menos entre el momento en que se le confirmó histopatológicamente la presencia de ésta enfermedad y el primer tratamiento.

En la Tabla I, se describen las características sociodemográficas en los pacientes cuya información estaba consignada en la HC. Sin distinción por sexo, el $74 \%$ vivían en Medellín y su área metropolitana, el $63 \%$ estaban casados o en unión libre, un poco más de la mitad pertenecían a estratos socioeconómicos bajos, casi dos terceras partes tenían niveles educativos $\leq$ primaria, aunque un $63 \%$ estaban afiliados al régimen contributivo en salud.

Las principales características clínicas de los pacientes con cáncer escamocelular oral se presentan en la Tabla II. Un $47 \%$ recibió combinación de tratamientos y a un $28 \%$ se le realizó solo Cirugía (sin diferencias estadísticamente significativas según sexo). Un $56 \%$ de las mujeres y un $63 \%$ de los hombres fueron diagnosticados en un estadio IV del tumor, presentándose diferencias estadísticamente significativas en la distribución porcentual según sexo y estadio $(p<0,0001)$. Un poco más de la tercera parte de los pacientes recibieron tratamiento para el dolor y cuidado paliativo y atención con nutricionista. Según las comorbilidades reportadas en la $\mathrm{HC}$, un $35 \%$ presentaron problemas cardiovasculares y un $9 \%$ problemas respiratorios. No se reportaron diferencias estadísticamente significativas entre la presencia de determinadas comorbilidades y el sexo. Se reportó el consumo de alcohol en un $19 \%$ y de cigarrillo en un $51 \%$ con mayor consumo en hombres en ambos casos y diferencias estadísticamente significativas $(p<0,0001)$ con respecto a las mujeres. En cuanto a complicaciones asociadas al tratamiento para el cáncer, se reportaron con mayor frecuencia disfagia (47 $\%)$, mucositis $(24 \%)$ y xerostomia $(20 \%)$. No se presentaron diferencias estadísticamente significativas según sexo en el reporte de las complicaciones asociadas al tratamiento.

De acuerdo a la distribución porcentual de la localización del tumor según sexo (Fig. 1), tanto para hombres como mujeres, más de la mitad de estos se presentaron en lengua. Se presentaron diferencias estadísticamente significativas $(p<0,05)$ en las frecuencias de presentación de los tumores en piso de boca, orofaringe, paladar duro y labio inferior. Por ultimo, atendiendo a la distribución porcentual de los tres tumores más frecuentes según el estadio en el cual fue- 
Tabla I. Características sociodemográficas de los pacientes con cáncer oral tratados en centros oncológicos de la ciudad de Medellín, Colombia.

\begin{tabular}{|c|c|c|}
\hline Características & $\mathrm{n}$ & $\%$ \\
\hline \multicolumn{3}{|l|}{ Sexo } \\
\hline Hombre & 422 & 54,2 \\
\hline Mujer & 356 & 45,8 \\
\hline Total & 778 & 100,0 \\
\hline \multicolumn{3}{|l|}{ Lugar de re sidencia } \\
\hline Medellín y sus corregimientos & 418 & 55,6 \\
\hline Municipios del Área Metropolitana & 140 & 18,6 \\
\hline Otros municipios de Antioquia & 142 & 18,9 \\
\hline Otros departamentos del país & 51 & 6,8 \\
\hline Total & 751 & 100,0 \\
\hline \multicolumn{3}{|l|}{ Estado civil } \\
\hline Soltero (a) & 134 & 20,4 \\
\hline Casado (a) & 352 & 53,5 \\
\hline Separado (a)/Divorciado (a) & 27 & 4,1 \\
\hline Unión libre & 62 & 9,4 \\
\hline Viudo (a) & 83 & 12,6 \\
\hline Total & 658 & 100,0 \\
\hline \multicolumn{3}{|l|}{ Nivel socioeconómico } \\
\hline Bajo & 88 & 56,0 \\
\hline Medio & 67 & 42.7 \\
\hline Alto & 2 & 1.3 \\
\hline Total & 157 & 100,0 \\
\hline \multicolumn{3}{|l|}{ Nivel educativo } \\
\hline Ninguno & 4 & 1,9 \\
\hline Primaria incompleta & 101 & 48,9 \\
\hline Primaria completa & 31 & 15,0 \\
\hline Básica secundaria incompleta & 18 & 8,7 \\
\hline Básica secundaria completa & 16 & 7,7 \\
\hline Técnica Tecnológica & 9 & 4,3 \\
\hline Universitaria & 28 & 13,5 \\
\hline Total & 207 & 100,0 \\
\hline \multicolumn{3}{|l|}{ Afiliación en salud } \\
\hline Contributivo & 465 & 63,1 \\
\hline Subsidiado & 212 & 28,8 \\
\hline Vinculado & 28 & 3,8 \\
\hline Particular & 32 & 4,3 \\
\hline Total & 737 & 100,0 \\
\hline
\end{tabular}

ron diagnosticados, se observa una tendencia en todos los casos a mayores frecuencias en estados más avanzados, con diferencias estadísticamente significativas según tipo de tumor y estadio, especialmente en mujeres cuando se hace análisis estratificado (Tabla III).

\section{DISCUSIÓN}

Los hallazgos principales de este estudio dan cuenta de 778 casos de CEB tratados por primera vez en 9 centros oncológicos de la ciudad de Medellín. En general, la edad de diagnóstico fue avanzada tanto en hombres como en mujeres. Una buena parte de estas personas recibieron diferentes combinaciones de tratamientos y llama la atención que la mayoría se diagnostican en estadios avanzados de la enfermedad. Se reportó mayor consumo de alcohol y cigarrillo en los hombres con CEB. Sepresentaron complicaciones asociadas a la enfermedad como por ejemplo disfagia, mucositis y xerostomia. La mayor parte de los tumores, se localizaron en lengua sin diferencias estadísticamente significativas por sexo. Este es uno de los primeros estudios que caracteriza en términos epidemiológicos, el CEB en Colombia, ante la ausencia de sistemas de información y vigilancia epidemiológica en el país para esta patología.

Un elemento importante a considerar es la edad promedio de diagnóstico de las personas en el presente estudio (64 años), el cual está en consonancia con otros estudios epidemiológicos (Scully \& Bagan, 2009; Álvarez Martínez et al.,

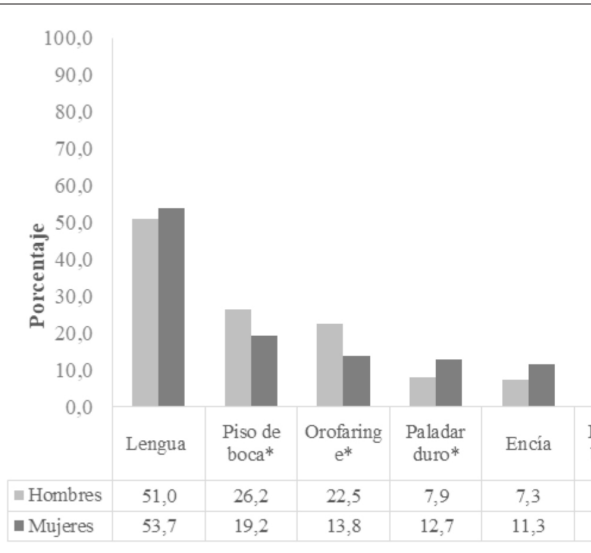

Fig. 1. Distribución porcentual de la localización del tumor en un solo sitio anatómico según sexo, en los pacientes con cáncer oral tratados en centros oncológicos, Medellín, Colombia.

* Pruebas Chi cuadrado para diferencia de proporciones $p<0,05$ 
POSADA-LÓPEZ, A.; PALACIO-CORREA, M. A. \& AGUDELO-SUÁREZ, A. A. Características Sociodemográficas y clínicas de los pacientes tratados por primera vez por cáncer escamocelular oral. Medellín, Colombia. Int. J. Odontostomat., 12(3):237-245, 2018.

Tabla II. Características clínicas de los pacientes con cáncer oral tratados en centros oncológicos de la ciudad de Medellín, Colombia.

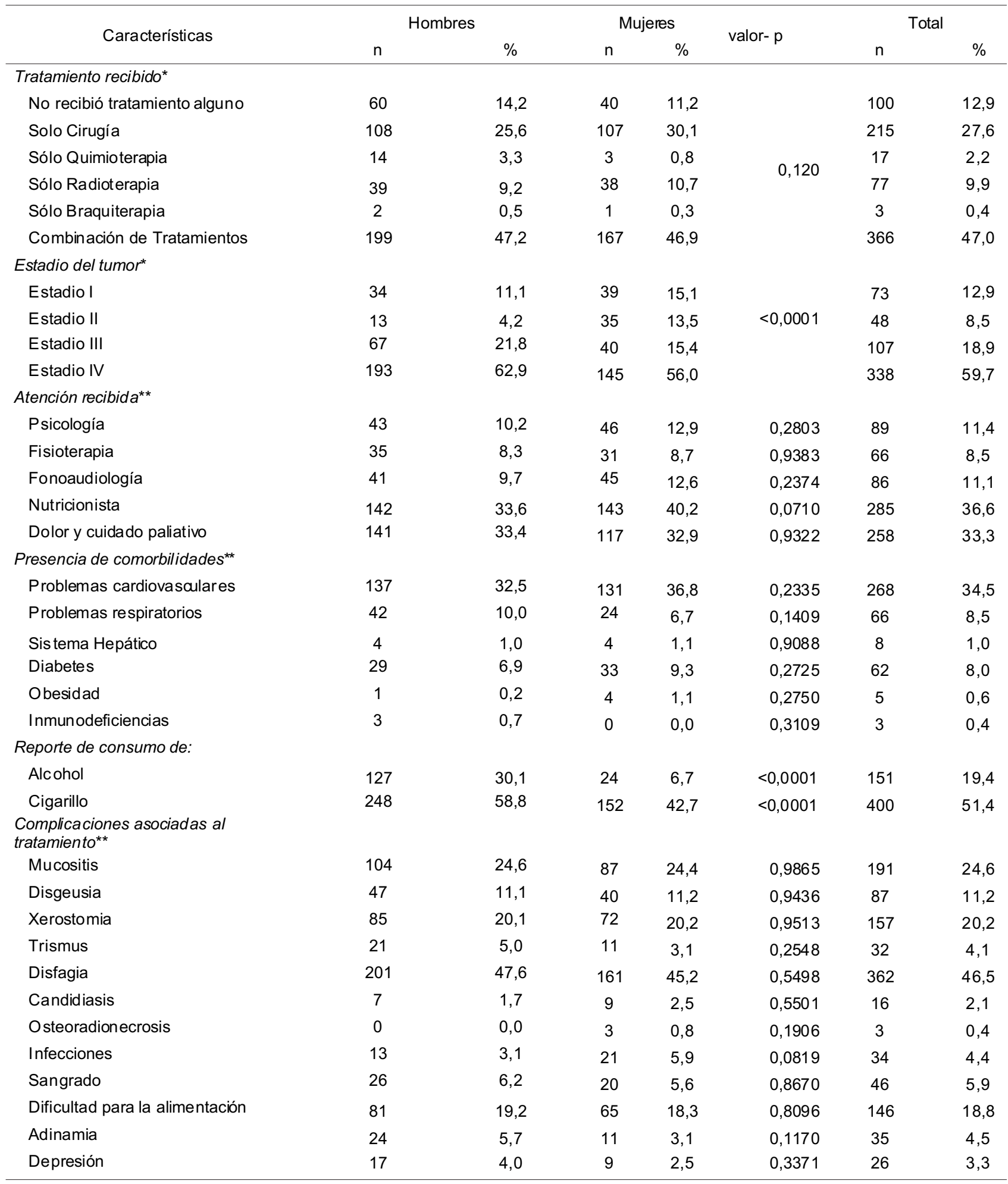

*pruebas Chi cuadrado de distribución de frecuencias ** Pruebas Chi cuadrado para diferencia de proporciones. 
POSADA-LÓPEZ, A.; PALACIO-CORREA, M. A. \& AGUDELO-SUÁREZ, A. A. Características Sociodemográficas y clínicas de los pacientes tratados por primera vez por cáncer escamocelular oral. Medellín, Colombia. Int. J. Odontostomat., 12(3):237-245, 2018.

Tabla III. Distribución de las tres localizaciones más frecuentes del tumor según estadio en los pacientes con cáncer bucal tratados en centros oncológicos, Medellín, Colombia.

\begin{tabular}{|c|c|c|c|c|c|c|}
\hline \multirow{2}{*}{ Localización del tumor } & \multicolumn{4}{|c|}{ Estadio del tumor $\mathrm{n}(\%)$} & \multirow{2}{*}{ Total } & \multirow{2}{*}{ Valor-p* } \\
\hline & Estadio I & Estadio II & Estadio III & Estadio IV & & \\
\hline \multicolumn{7}{|l|}{ Hombres } \\
\hline Lengua & $18(10,9)$ & $8(4,8)$ & $41(24,8)$ & $98(59,4)$ & 165 & \multirow{3}{*}{0,1331} \\
\hline Piso de la cavidad oral & $5(6,0)$ & $4(4,8)$ & $15(17,9)$ & $60(71,4)$ & 84 & \\
\hline Orofaringe & $3(4,2)$ & $1(1,4)$ & $13(18,3)$ & $54(76,1)$ & 71 & \\
\hline \multicolumn{7}{|l|}{ Mujeres } \\
\hline Lengua & $25(16,4)$ & $19(12,5)$ & $22(14,5)$ & $86(65,6)$ & 152 & \multirow{3}{*}{0,0260} \\
\hline Piso de la cavidad oral & $6(12,0)$ & $7(14,0)$ & $1(2,0)$ & $36(72,0)$ & 50 & \\
\hline Orofaringe & $1(2,6)$ & $2(5,3)$ & $5(13,2)$ & $30(78,9)$ & 38 & \\
\hline \multicolumn{7}{|l|}{ Total } \\
\hline Lengua & $43(13,6)$ & $27(8,5)$ & $63(19,9)$ & $184(58,0)$ & 317 & \multirow{3}{*}{0,0015} \\
\hline Piso de la cavidad oral & $11(8,2)$ & $11(8,2)$ & $16(11,9)$ & $96(71,6)$ & 134 & \\
\hline Orofaringe & $4(3,7)$ & $3(2,8)$ & $18(16,5)$ & $84(65,0)$ & 109 & \\
\hline
\end{tabular}

${ }^{*}$ Pruebas chi cuadrado de distribución de frecuencias.

2011). El cáncer de la cavidad oral es una enfermedad crónica que se diagnostica con mayor frecuencia en edades más avanzadas haciendo la salvedad de que puede presentarse en edades tempranas (Modh et al., 2018). En esto intervienen factores propios del contexto demográfico y epidemiológico del país, caracterizado por un el aumento de la esperanza de vida y su consecuente aumento de la población adulta mayor, lo cual conlleva al aumento de las enfermedades crónicas y degenerativas propias de países de mediana o alta renta (Lence \& Camacho, 2006; Ramos-Clason 2012). Como dato relevante, se encuentra la presentación de un caso de CEB en un niño de 7 años. Una revisión de la literatura de artículos publicados entre 1936 y 2012 reportó 186 casos de CEB en menores de 20 años (Bodner et al., 2014) y 13 casos ocurrieron en población infantil de 7 años o menos. Aunque no son casos frecuentes, se deben explorar con mayor profundidad los factores de riesgo y los determinantes de orden social y familiar que pueden influir en la presentación del CEB en población infantil y así establecer estrategias de diagnóstico y planes de tratamiento oportunos y menos invasivos.

Otro aspecto a considerar en los hallazgos encontrados tiene que ver con las posibles variaciones en el tiempo trascurrido entre la percepción de la lesión por parte del paciente (o su respectiva consulta con el profesional), la confirmación del diagnóstico de CEB y su inicio de tratamiento. Si bien en muchos casos no fue posible tener información en las historias clínicas de los pacientes, se pueden inferir posibles explicaciones relacionadas con los posibles determi- nantes del acceso a los servicios de salud en el Sistema General de Seguridad Social en Salud para Colombia (Vargas \& Molina, 2009; Rocha-Buelvas, 2013). Un estudio realizado en Medellín sobre la alteraciones orales en pacientes sometidos a radioterapia por Cáncer de cabeza y cuello (Álvarez Gómez et al., 2017), puso en manifiesto posibles barreras asistenciales a los servicios de salud que impiden un diagnóstico y tratamiento oportuno por parte de los profesionales, más otras barreras que son estructurales y características del sistema de atención en salud. De igual manera se evidenció en los resultados del estudio en Medellín que la mayoría de los tumores se diagnosticaron en estadios más avanzados y esto reafirma algunos problemas de acceso a programas de cribado y de prevención para Cáncer Oral oportunos (Buelvas \& Agudelo, 2011).

Respecto al sexo, hombres y mujeres reportaron una proporción similar de CEB en el presente estudio, aunque existen investigaciones que indican que la proporción de hombres con CEB es mayor que en las mujeres y puede ser explicado este fenómeno por las actividades laborales de los hombres (Guzmán et al., 2011). No obstante, se encuentra otros estudios donde se menciona que razón entre hombres y mujeres es muy similar, y que puede relacionarse con los comportamientos sociales que han incrementado en la mujer el consumo de cigarrillo y alcohol (Scully \& Bagan, 2009; Gaitán Cepeda et al., 2011). Un elemento a contrastar tiene relación con el hecho de que en nuestro estudio, se encontró mayor frecuencia de consumo de alcohol y cigarrillo en los pacientes hombres diagnosticados con CEB. 
Los pacientes de este estudio fueron en su mayoría procedentes y residentes del área urbana de Medellín y afiliados al régimen contributivo (personas con capacidad de pago), aunque algunos en el momento de la prestación del servicio no tenían ninguna cobertura en salud. También reportaron niveles socioeconómicos y educativos más bajos. Dicha situación es soportada con varios estudios que concuerdan como existen determinantes sociales y estructurales que pueden afectar la frecuencia y distribución del Cáncer Oral (Buelvas \& Agudelo; Dourado Martins et al., 2014). Por ello, se requieren más y mejores estudios que incluyan variables sociales y contextuales, así como el estudio de la construcción social del proceso salud enfermedad que den cuenta de factores culturales y de autocuidado involucrados en la aparición del CEB en diferentes grupos sociales.

En general, la literatura reporta que el cáncer oral es diagnosticado en estadios avanzados, información que coincide con losdatos de los pacientes evaluados (Petti \& Scully, 2007; Scully \& Bagan, 2007). Se encontró que la lesión tumoral estaba localizada en un solo lugar de la boca, siendo la lengua la localización más frecuente, seguido por piso de boca y, orofaringe y en estas dos últimas con diferencias significativas por sexo. Los valores concuerdan con un estudio previo realizado en la ciudad (Álvarez Martínez et al., 2011). Los tratamientos más frecuentes para estos pacientes fueron aquellos combinados y cirugía. Esto es importante analizar ya que en una buena parte de los casos, se afecta la fisionomía y la fonación de las personas y puede llegar a ser un tratamiento agresivo, invasivo y mutilante, afectando la calidad de vida de las personas y de su entorno familiar y social inmediato (Chandu et al., 2006). Por ello importante que el paciente reciba apoyo adicional al tratamiento principal como la atención interdisciplinaria por nutricionista, fisioterapia y fonoaudiología, los cuales fueron reportados en la historia clínica de los pacientes atendidos en el periodo evaluado.

Se encontraron algunas complicaciones asociadas al tratamiento de los pacientes con CEB las cuales están reportadas en la literatura (Jham \& da Silva Freire, 2006; Álvarez Gómez et al., 2017). En el periodo evaluado fueron más frecuentes mucositis, xerostomía y disfagia y en general sin diferencias significativas entre hombres y mujeres. Ofrecer un tratamiento integral, requiere del manejo interdisciplinario entre los especialistas en cirugía oral y maxilofacial y los odontólogos generales, estos últimos deben contar con la formación y capacitación necesaria en aspectos fundamentales sobre el diagnóstico y tratamiento oportuno del CEB y el manejo de sus posibles complicaciones asociadas.

En la interpretación de los resultados es importante comentar aquellas limitaciones relacionadas con su metodología. El análisis de las variables del estudio dependen de lo recolectado en la historia clínica y se evidenció que una buena parte de ellas no tenía información sociodemográfica y clínica importante para los objetivos del estudio. Por ello se evidencia la necesidad de fortalecer los sistemas de vigilancia epidemiológica para la investigación y la acción en salud. Una mala clasificación de la enfermedad según la CIE-10 por parte de las instituciones, genera un sesgo de información que el investigador no puede controlar. Como fortalezas, destacar que es un estudio de amplio alcance al incluir 9 instituciones que atienden pacientes con CEB. La asesoría temática de un cirujano oncólogo de cabeza y cuello permitió controlar posibles sesgos de observación, especialmente en lo que se relaciona con las variables clínicas.

Se requieren nuevas investigaciones que den cuenta de otras variables relacionadas con la frecuencia y distribución del CEB, desde modelos multivariados y multinivel. De igual manera, conviene estudiar otros elementos como por ejemplo la supervivencia del CEB y la calidad de vida de los pacientes y del entorno familiar, este último ítem a través de otras metodologías desde la epidemiología social y los estudios cualitativos.

Como conclusión se encontraron diferentes características sociodemográficas y clínicas relacionadas con el CEB que merecen atención y seguimiento oportunos. Se evidencia la necesidad de programas que permitan el diagnóstico y tratamiento oportuno de esta enfermedad, así como la articulación de estrategias locales a otras nacionales y globales como por ejemplo el al Plan Nacional de Lucha Contra el Cáncer 2010-2019 para Colombia y otros planes a nivel global propuestos desde organismos internacionales como la Organización Panamericana y Mundial de la Salud.

\section{AGRADECIMIENTOS}

A los profesores de la Facultad Nacional de Salud Pública: Hugo Grisales, Carolina Salas Zapata, Juan Fernando Saldarriaga, María Patricia Arbeláez, por sus asesorías durante la realización de la investigación. A los Especialistas Sergio Fabián Zúñiga Pavía y Efraín Álvarez Martínez por su asesoría temática. 
POSADA-LÓPEZ, A.; PALACIO-CORREA, M. A. \& AGUDELO-SUÁREZ, A. A. Sociodemographic and Clinic Characteristics of the Patients Threatened at First Time for Squamous Cell Oral Cancer. Medellín (Colombia). Int. J. Odontostomat., 12(3):237-245, 2018.

ABSTRACT: This study aimed to describe the sociodemographic and clinical characteristics of patients treated for first-time oral squamous cell carcinoma (OSCC), in Medellín (Colombia). For this purpose, a descriptive study was carried out within a retrospective dynamic cohort of OSCC cases from 9 specialized oncology centers in the city between 2000 and 2011, according to the information of the clinical records and classified according to the ICD-10. Sociodemographic characteristics were recorded and clinical variables were collected such as: Treatment received, tumor location, tumor stage, care received (professional), report of alcohol or cigarette consumption, complications associated with OSCC treatment. The study variables were described, and Chi square test was calculated in order to observe differences between some clinical characteristics and sex. In the study period 778 cases of OSCC were found, with an average age of diagnosis of $63.5( \pm 13.6)$ years. More than half had low socioeconomic and educational levels. Fifty six percent of women and $63 \%$ of men were diagnosed in stage IV of the tumor. Treatments performed most, were either combined or for surgery. Thirty five percent of the cases had cardiovascular problems, although no statistically significant differences were reported between the presence of certain comorbidities and sex. Alcohol consumption was reported in $19 \%$ and smoking in $51 \%$, with higher consumption in men in both cases and statistically significant differences ( $p$ $<0.0001$ ) with respect to women. Regarding complications associated with treatment for OSCC, dysphagia (47\%) and mucositis $(24 \%)$ were reported more frequently. More than half of the tumors were presented in the tongue. Strategies are required that allow epidemiological surveillance systems for the OSCC, as well as to improve prevention and early treatment programs for this disease in line with national and global policies and plans.

KEY WORDS: carcinoma, squamous cell, mouth neoplasms, health profile, epidemiological monitoring

\section{REFERENCIAS BIBLIOGRÁFICAS}

Álvarez Gómez, G. J.; Alvarez Martínez, E.; Jiménez Gómez, R.; Mosquera Silva, Y.; Gaviria Núñez, A. M.; Garcés Agudelo, A.; Alonso Duque, A.; Zabala Castaño, A.; Echeverri González, E.; Isaac Millán, M. \& Ramírez Ossa, D. Reverse smokers's andchanges in oral mucosa. Department of Sucre, Colombia. Med. Oral. Patol. Oral Cir. Bucal, 13(1):E1-E8, 2008.

Álvarez Gómez, G. J.; López Camacho, R. V.; Botero Torres, J. E.; Botero Gómez, S. M.; Cardona Alzate, D. P.; Carmona Ross, P. A. \& Hernández Arévalo, J. E. Alteraciones en la cavidad bucal en pacientes tratados con radioterapia de cabeza y cuello. Medellín, Colombia. Rev. Odontol. Mex., 21(2):87-97, 2017.
Álvarez Martínez, E.; Preciado Uribe, A.; Montoya Fernández, S.; Jiménez Gómez, R. \& Posada López, A. Características clínicas e histopatológicas del carcinoma escamocelular bucal en el periodo 1990-2004 en Medellín, Colombia. Rev. Cuba. Estomatol., 48(4):320-9, 2011

Álvarez Martínez, E.; Preciado, U. A.; Montoya Fernández, S. A.; Jiménez Gómez, R. \& Posada López, A. Características clínicohistopatológicas del carcinoma escamocelular bucal, Colombia. Rev. Cuba. Estomatol., 47(1):81-95, 2010.

Bernal-Baláez, A. E. Estudio epidemiológico del cáncer bucal en Colombia 1989-2008. Rev. Fac. Med., 64(1):75-8, 2016.

Bodner, L.; Manor, E.; Friger, M. D. \& van der Waal, I. Oral squamous cell carcinoma in patients twenty years of age or younger--review and analysis of 186 reported cases. Oral Oncol., 50(2):84-9, 2014

Buelvas, A. R. \& Agudelo, A. A. Gradiente social, envejecimiento y diagnóstico tardío del cáncer oral. Rev. Fac. Nac. Salud Pública, 29(3):320-8, 2011.

Chandu, A.; Smith, A. C. \& Rogers, S. N. Health-related quality of life in oral cancer: a review. J. Oral Maxillofac. Surg., 64(3):495502, 2006.

Colombia Departamento Administrativo de Planeación. Preguntas frecuentes de estratificación. Perfil Socioeconómico. Medellín Total. Medellín, Departamento Administrativo de Planeación, 2011.

Dourado Martins, J.; Oliveira Mascarenhas Andrade, J.; Souza Freitas, V. \& de Araújo, T. M. Social determinants of health and the occurrence of oral cancer: a systematic literature review. Rev. Salud Publica (Bogota), 16(5):786-98, 2014

Ferlay, J.; Soerjomataram, I.; Dikshit, R.; Eser, S.; Mathers, C.; Rebelo, M.; Parkin, D. M.; Forman, D. \& Bray, F. Cancer incidence and mortality worldwide: sources, methods and major patterns in GLOBOCAN 2012. Int. J. Cancer, 136(5):E359-86, 2015.

Gaitán Cepeda, L. A.; Peniche Becerra, A. G. \& Quezada Rivera, D. Tendencias temporales de frecuencia y prevalencia de cáncer oral y carcinoma de células escamosas oral en Mexicanos. Un estudio retrospectivo de 20 años. Med. Oral Patol. Oral Cir. Bucal, 16(4):205-9, 2011.

Guerrero, R.; Gallego, A. I.; Becerril-Montekio, V. \& Vásquez, J. Sistema de salud de Colombia. Salud Publica Mex., 53(Supl. 2):s144-55, 2011

Guzmán, G. P.; Villaseca, H. M.; Antonio, P. L.; Araya, O. J.; Aravena, M. P.; Cravero, P. C.; Pino, M. P. \& Roa, S. J. Carcinoma epidermoide oral y orofaríngeo. Estudio clínico-patológico. Rev. Chil. Cir., 63(3):250-6, 2011.

Instituto Nacional de Cancerología. Cáncer en Cifras. Bogotá, Instituto Nacional de Cancerología, Ministerio de Salud y Protección Social, Colombia, 2017. Disponible en: http://www.cancer.gov.co/ cancer en cifras.

Jham, B. C. \& da Silva Freire, A. R. Oral complications of radiotherapy in the head and neck. Braz. J. Otorhinolaryngol., 72(5):704-8, 2006.

Lence, J. J. \& Camacho, R. Cáncer y transición demográfica en América Latina y el Caribe." Rev. Cuba. Salud Publica, 32(3), 2006.

Martínez Martínez, A.; Carmona Lorduy, M. \& Diaz-Caballero, A. Comportamiento del cáncer bucal en un hospital de Cartagena de Indias, Colombia. Rev. Cuba. Estomatol., 53(2):24-8, 2016.

Ministerio de Salud y Protección Social. Resolución nº 008430 de 1993 (4 de octubre de 1993). Por la cual se establecen las normas científicas, técnicas y administrativas para la investigación en salud. Bogotá, Ministerio de Salud y Protección Social, Colombia, 1993.

Modh, A.; Gayar, O. H.; Elshaikh, M. A.; Paulino, A. C. \& Siddiqui, F. Pediatric head and neck squamous cell carcinoma: Patient demographics, treatment trends and outcomes. Int. J. Pediatr. Otorhinolaryngol., 106:21-5, 2018. 
Ordóñez, D.; Aragón, N.; García, L. S.; Collazos, P. \& Bravo, L. E. Cáncer oral en Santiago de Cali, Colombia: análisis poblacional de la tendencia de incidencia y mortalidad. Salud Publica Mex., 56(5):465-72, 2014.

Petti, S. \& Scully, C. Oral cancer knowledge and awareness: primary and secondary effects of an information leaflet. Oral Oncol., 43(4):408-15, 2007.

Posada-López, A.; Palacio, M. A.; Salas, C.; Álvarez, E. \& Grisales, $\mathrm{H}$. Supervivencia de los pacientes con cáncer escamocelular bucal, tratados por primera vez, en centros oncológicos en el periodo 2000 a 2011, Medellín-Colombia. Rev. Fac. Odontol. Univ. Antioq., 27(2):245-61, 2016.

Ramos-Clason, E. C. Transición epidemiológica en Colombia: de las enfermedades infecciosas a las no transmisibles. Rev. Cienc. Biomed., 3(2):282-90, 2012.

Rocha-Buelvas, A. Análisis sobre el acceso a los servicios de la salud bucal: un indicador de equidad. Rev. Gerenc. Polit. Salud, 12(25):96-112, 2013.

Scully, C. \& Bagan, J. Oral squamous cell carcinoma: overview of current understanding of aetiopathogenesis and clinical implications. Oral Dis., 15(6):388-99, 2009.

Scully, C. \& Bagan, J. V. Recent advances in Oral Oncology. Oral Oncol., 43(2):107-15, 2007.

Shield, K. D.; Ferlay, J.; Jemal, A.; Sankaranarayanan, R.; Chaturvedi, A. K.; Bray, F. \& Soerjomataram, I. The global incidence of lip, oral cavity, and pharyngeal cancers by subsite in 2012. CA Cancer J. Clin., 67(1):51-64, 2017.

Vargas, J. J. \& Molina, M. G. Acceso a los servicios de salud en seis ciudades de Colombia: limitaciones y consecuencias. Rev. Fac. Nac. Salud Pública, 27(2):121-30, 2009.

von Elm, E.; Altman, D. G.; Egger, M.; Pocock, S. J.; P. C. Gøtzsche and J. P. Vandenbroucke (2008). The Strengthening the Reporting of Observational Studies in Epidemiology [STROBE] statement: guidelines for reporting observational studies. Gac. Sanit., 22(2):144-50, 2008.

World Medical Association General Assembly. World Medical Association Declaration of Helsinki: ethical principles for medical research involving human subjects. J. Int. Bioethique, 15(1):1249, 2004.

\author{
Dirección para correspondencia: \\ Adriana Posada López \\ Facultad de Odontología \\ Universidad de Antioquia \\ Calle $70 \mathrm{~N}^{\circ} 52-21$ \\ Medellín \\ COLOMBIA
}

Email: adriposalo@gmail.com

Recibido : 11-05-2018

Aceptado: 07-06-2018 